\title{
Gas sensing using polymer-functionalized deformable Fabry-Perot interferometers
}

\author{
Raphael St-Gelais ${ }^{\mathrm{a}}$, Gillian Mackey ${ }^{\mathrm{b}}$, John Saunders ${ }^{\mathrm{b}}$, Jingjing Zhou ${ }^{\mathrm{b}}$, Antoine Leblanc-Hotte ${ }^{\mathrm{a}}$, \\ Alexandre Poulin ${ }^{a}$, Jack A. Barnes ${ }^{b}$, Hans-Peter Loock ${ }^{b}$, R. Stephen Brown ${ }^{b}$, Yves-Alain Peter ${ }^{a, *}$ \\ a Department of Engineering Physics, Polytechnique Montréal, P.O. Box 6079, Station Centre-Ville, Montréal, QC, H3C 3A7, Canada \\ ${ }^{\mathrm{b}}$ Department of Chemistry, Queen's University, Kingston, ON, K7L 3N6, Canada
}

\section{A R T I C L E I N F O}

Article history:

Received 1 October 2012

Received in revised form

31 December 2012

Accepted 4 February 2013

Available online $\mathrm{xxx}$

Keywords:

Gas sensing

Fabry-Perot interferomer

Polymer swelling

Volatile organic compound

Silicon micromachining

\begin{abstract}
A B S T R A C T
We report a chemical vapor sensor in which polymer swelling, upon analyte absorption, is used to deform an on-chip silicon Fabry-Perot interferometer (FPI). The magnitude of the deformation, recorded through the resonance wavelength shift, is proportional to the analyte concentration in accordance with a simplified analytical model and with finite element simulations. Conventional and phenyl-doped polydimethylsiloxane (PDMS) polymers are used to functionalize different interferometers, which are tested for the detection of two volatile organic compounds, i.e. $\mathrm{m}$-xylene and cyclohexane. The detection of $\mathrm{m}$-xylene concentrations down to $34 \mathrm{ppm}$-limited by our flow-meter setup-is achieved experimentally. Based on the sensitivities and the noise characteristics of the devices, limits of detection (LODs) of $1.6 \mathrm{ppm} \mathrm{m}$-xylene and $6.3 \mathrm{ppm}$ cyclohexane are expected.
\end{abstract}

(c) 2013 Elsevier B.V. All rights reserved.

\section{Introduction}

Upon analyte absorption, many polymers (e.g., PDMS) are known to expand [1], a phenomenon usually referred to as "polymer swelling". Such expansion can be used to deform optical interferometers, causing a shift of their resonance condition that can be monitored to create chemical sensors. This approach was previously used with various optical interferometers. Polymercoated fiber Bragg gratings were shown to deform upon exposition to salt [2] hydrocarbons [3] and humidity [4]. Bragg gratings were also fabricated directly with deformable polymer layers and were found to be sensitive to the presence of acetone [5], salt [6], and volatile organic compounds [7] (VOCs). Fabry-Perot interferometers (FPI) can also be formed simply by exploiting reflections at the two material interfaces of single deformable polymer membranes. This approach was used to detect VOCs [8-10] and ionic strength [11].

The sensors reported here rely on deformable Fabry-Perot interferometers. As presented in Fig. 1, these FPIs are implemented in-plane with the substrate by vertical plasma etching of siliconair Bragg reflectors. The in-plane (i.e.: vertical mirrors, in-plane

\footnotetext{
* Corresponding author. Tel.: +1 514340 4711x3100; fax: +1 5143405195

E-mail addresses: yves-alain.peter@polymtl.ca, yap@ieee.org (Y.-A. Peter).
}

optical axis) rather than out-of-plane [5-11] configuration allows monolithic integration of microfluidic systems and optical fiber alignment grooves. Integrated microfluidic systems simplify the parallel functionalization of multiple interferometers, using different polymers, to target specific classes of analytes. Fiber grooves allow the remote interrogation of the interferometers with passively aligned conventional single-mode optical fibers, rather than requiring free space alignment of light sources and photodetectors [5-11].

\section{Methodology}

\subsection{Sensing mechanism}

Upon analyte absorption, three factors can cause shifts of the interferometer $\left(\Delta \lambda_{\text {Res }}\right)$ resonance wavelength. First, as depicted in Fig. 1(b), polymer swelling deforms the interferometer, resulting in an increase $(\Delta L)$ of the mirror separation $(L)$. This mechanism will later be demonstrated to be the dominant one. Secondly, if the refractive index of the analyte $\left(n_{a}\right)$ and the polymer $\left(n_{p}\right)$ are different, the refractive index of the polymer-analyte mixture $(n)$ should change by a factor $\Delta n_{\text {Mix }}$ upon sample absorption. Lastly, if the mirrors oppose a significant force to the polymer expansion, there should be a densification of the polymer, which should lead to a $\Delta n_{\sigma}$ variation of its refractive index. All three effects change 


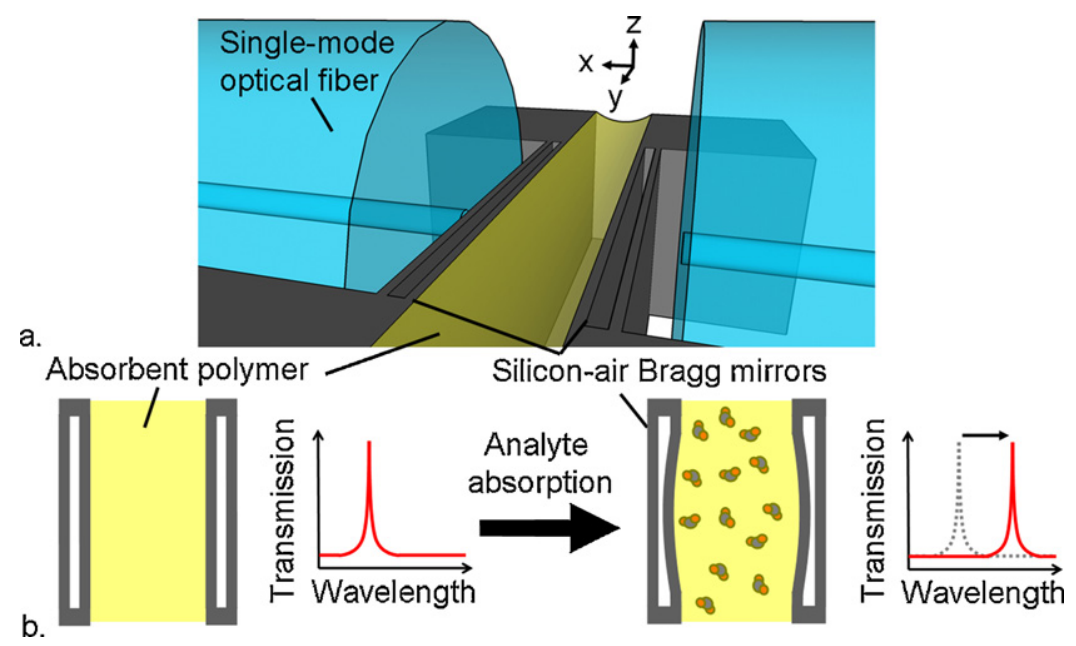

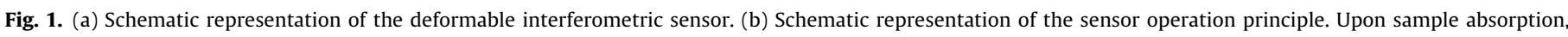

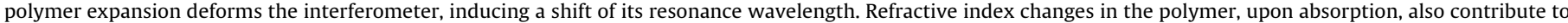
this shift.

the interferometer optical pathlength $(n L)$, and contribute to the measured resonance wavelength shift $\left(\Delta \lambda_{\text {Res }}\right)$ through:

$\frac{\Delta \lambda_{\text {Res }}}{\lambda_{\text {Res }}}=\alpha \frac{\Delta L}{L}+\beta \frac{\left(\Delta n_{\mathrm{Mix}}+\Delta n_{\sigma}\right)}{n}$,

where $\alpha=0.75$ and $\beta=0.93$ are device-specific reduction factors $(\leq 1)$. The factor $\alpha$ accounts for the fact that only one layer in each Bragg mirror is displaced upon analyte absorption (see Fig. 1b). The factor $\beta$ accounts for the fact that the resonant mode inside the cavity is not located entirely inside the polymer, but also partly inside the mirrors (i.e., the thickness of the mirrors is not negligible compared to $L$, the distance between the mirrors). The value of both parameters can be calculated using a previously reported optical simulation algorithm for deep-etched Fabry-Perot interferometers [12], as detailed in Appendix A1.

We define the dimensionless sensitivity $\left(\Gamma_{\phi}\right)$ as the normalized ratio of the resonance wavelength shift $\left(\Delta \lambda_{\text {Res }}\right)$ to the absorbed volume fraction of analyte $\left(\phi_{a}\right)$ inside the polymer:

$\Gamma_{\phi}=\frac{1}{\lambda_{\operatorname{Res}}} \frac{\Delta \lambda_{\text {Res }}}{\Delta \phi_{a}}$

Conveniently, $\phi_{a}$ can be related to the volume/volume (v/v) concentration of analyte in air $\left(C_{\text {air }}\right)$, near the polymer, using the ideal gas law and the partition coefficient of the polymer for the respective analyte $\left(K_{p-a}\right)$ :

$\phi_{a}=K_{p-a} \frac{P V_{\mathrm{m}}}{R T} C_{\mathrm{air}}$

where $P$ is the pressure (1 atm in our case), $T$ is the temperature, $R$ is the gas constant, and $V_{\mathrm{m}}$ is the molar volume of the analyte, in liquid phase.

For all the calculations presented in this work we assume that upon absorption, the final volume of the polymer-analyte mixture equals the initial volume of the polymer, plus the absorbed volume of analyte. In other words we assume that there is no volume reduction upon absorption, except in the presence of mechanical stresses inside the polymer (calculations in Section 2.1.1 will demonstrate these stresses lead to negligible volume reductions). In this context, the relative volume $(V)$ expansion of the polymer upon absorption of a $\phi_{a}$ volume fraction of analyte is given by:

$\frac{\Delta V}{V}=\Delta \phi_{a}$

This assumption of volume additivity is supported by reports that the absorption of a slightly lower refractive index analyte (cyclohexane) reduced the refractive index of a PDMS-based polymer [13]. This would not have been possible if significant volume reduction occurred upon absorption, since volume reduction would have led to an increase of the refractive index.

\subsubsection{Simplified analytical model}

This section details the sensitivity $\left(\Gamma_{\phi}\right)$ of deformable FPI sensors in the case where:

(1) the mirrors are perfectly movable, such that they oppose negligible forces to the expansion of the polymer,

(2) the volume increase of the polymer (i.e. swelling) is directed mainly in one direction, parallel to the optical pathlength between the mirrors $(L)$, such that $\Delta L$ is maximized.

Interestingly, these conditions straightforwardly apply to outof-plane deformable Fabry-Perot interferometers [8-11]. In these cases, the polymer is bonded to a rigid substrate such that swelling can only occur in the out-of-plane direction, which is parallel to the optical axis (criterion 2). There is also no force opposing the movement of the top material interface that is used as the movable mirror of the interferometer (criterion 1 ).

For the in-plane devices presented in the current work (Fig. 1), compliance with the two above mentioned criteria is less trivial. The deformable layer in each Bragg mirror might oppose nonnegligible force to the expansion of the polymer. The polymer might also swell upward (positive $z$ direction in Fig 1a) if the bonding strength with silicon is not strong enough. We will therefore use the simplified ideal formalism presented in this section primarily as a basis for comparison of our in-plane sensor with out-of-plane sensing mechanisms. In Section 2.1.2 finite element simulations will address the limitations of the analytical model for in-plane devices.

Under the two conditions listed above, it is possible to show that the relative length increase between the mirrors, upon absorption of a $\phi_{a}$ volume fraction of analyte, is given by:

$$
\left.\frac{\Delta L}{L}\right|_{\text {Simplified }}=\frac{\Delta \phi_{a}}{3} \frac{1+v}{1-v}
$$

where $v$ is the polymer Poisson's ratio. It is also possible to show that the compressive stress experienced by the polymer due to its 
restrained expansion in the directions perpendicular to the optical axis ( $y$ and $z$ in Fig. 1 ) is given by:

$\sigma_{\perp}=\frac{\phi_{a} E}{3(1-v)}$

where $E$ is the Young's modulus of the polymer. This stress will in turn lead to a volume reduction of the polymer given by:

$\frac{\Delta V_{h}}{V}=\frac{\Delta \sigma_{h}}{B}=\frac{2}{9} \frac{\Delta \phi_{a} E}{B(1-v)}$

where $B$ is the bulk modulus of the polymer, and $\sigma_{h}=2 / 3 \sigma_{\perp}$ is the hydrostatic stress inside the polymer.

Also, the addition of a $\phi_{a}$ analyte volume fraction will lead to a $\Delta n$ variation of the polymer refractive index. Several rules exist to predict the refractive index of binary mixtures $[14,15]$. The Lorentz-Lorenz relation is probably most frequently used. We however choose to use the simpler Gladstone-Dale relation, which yields very similar results for the typical refractive indices that we will encounter. This relation (Eq. (8)) is linear relative to $\phi_{a}$, leading to a simplified expression of the sensitivity $\left(\Gamma_{\phi}\right)$.

$\frac{\Delta n_{\mathrm{Mix}}+\Delta n_{\sigma}}{n}=\Delta \phi_{a} \frac{\left(n_{a}-n_{p}\right)}{n_{p}}+\frac{\Delta V_{h}}{V} \frac{\left(n_{p}-1\right)}{n_{p}}$

Finally, combining Eqs. (1, 5-8) in Eq. (2) yields the sensitivity of deformable Fabry-Perot chemical sensors in the context of the simplified analytical model:

$\left.\Gamma_{\phi}\right|_{\text {Simplified }}=\frac{\alpha}{3} \frac{1+v}{1-v}+\beta \frac{\left(n_{a}-n_{p}\right)}{n_{p}}+\beta \frac{2}{9} \frac{\left(n_{p}-1\right)}{n_{p}} \frac{E / B}{1-v}$

In Eq. (9a), the first term accounts for interferometer deformations. The second term accounts for polymer refractive index variations due to mixture with an analyte of different refractive index. The third term accounts for refractive index variations due to hydrostatic stress upon expansion.

The absorbent polymers used in the following experiments are essentially incompressible. For example, the bulk modulus [16] $\left(B=10^{3} \mathrm{MPa}\right)$ of Sylgard $184 \mathrm{PDMS}$ is significantly higher than its Young's modulus [17] ( $E=1.8 \mathrm{MPa})$, which yield a $v=0.5-E / 2 B=0.499$ Poisson's ratio. In this context, the third term of Eq. (9a) is almost 3 orders of magnitude smaller than the first two terms and can be neglected. Eq. (9a) can therefore be rewritten as:

$$
\left.\Gamma_{\phi}\right|_{\text {Simplified }} \approx \alpha+\beta \frac{\left(n_{a}-n_{p}\right)}{n_{p}}
$$

Furthermore, for most organic compounds, the refractive index difference in Eq. (9b) $\left(n_{a}-n_{p}\right)$ should be in the order of 0-0.15. In these cases, the term that accounts for interferometer deformations $(\alpha)$ should always be at least 10 times larger than the term that accounts for refractive index variations. Therefore, with $\alpha=0.75$, the absorption of, for example, a $\phi_{a}=1 \%$ volume fraction of analyte is expected to induce a $\Delta \lambda_{\text {res }} / \lambda_{\text {res }} \approx 0.75 \%$ shift of the interferometer resonance wavelength.

\subsubsection{Finite element simulations}

In order to evaluate if the response of the fabricated devices can be expected to be close to the simplified analytical case, finite element simulations are performed using CoventorWare 2010. An isotropic expansion of the polymer volume filling the gap between the two mirrors (see Fig. 1) is simulated. Upon expansion, the relative increase of the distance between the mirrors $(\Delta L / L)$ and the hydrostatic pressure inside the polymer $\left(\sigma_{h}\right)$ are evaluated. For these simulations, we use the Young's modulus ( $E=1.8 \mathrm{MPa}$ ) [17] and Poisson's ratio ( $v=0.499)$ values of Sylgard 184 PDMS.

The meshed model used for the simulations is presented in Fig. 2(a). For each of the two Bragg mirrors, only the one silicon layer that is in contact with the polymer is included in the model.
The $(y, z)$ dimensions of these layers are $(130 \mu \mathrm{m}, 80 \mu \mathrm{m})$. Their thickness (in the $x$ direction) is $600 \mathrm{~nm}$, as determined by previous optical characterization of the interferometers [12]. The layers are attached at both $y$ extremities to the edges of the microfluidic channel, which are considered to be immobile. The bottom $z$ extremities of the mirror layers are fixed to the substrate (Fix All boundaries in Fig. 2a) while their upper $z$ extremities are free to move.

Two different types of boundary conditions are used to simulate the contact interfaces between silicon and PDMS. The first type considers the two materials to be tightly bonded together, such that no sliding can occur. The second type considers the two materials to be in contact but to be free to slide on each other without friction. We expect the experimental results to lie somewhere between these two extreme cases, which we will refer to as the "bonded" and "sliding" models.

Only a small portion of the microfluidic channel length $(40 \mu \mathrm{m})$ is included in the mesh model on each side of the Fabry-Perot cavity. The continuation of the truncated channel must therefore be simulated using appropriate boundary conditions. It is possible to show that, away from the deformable mirror layers, no polymer movement occurs in the directions $(x, y)$ parallel to the substrate due to the rigid boundaries of the microfluidic system. The continuation of the channels is therefore simulated using "Fix $Y$ " boundary conditions (see Fig. 2a).

For the two models, the response to various polymer volume increases is simulated. A linear relation is established between the absorbed volume fraction of analyte $\left(\phi_{a}\right)$ and the relative pathlength increase between the mirrors (on the optical axis of the system):

$$
\begin{aligned}
& \left.\frac{\Delta L}{L}\right|_{\text {Bonded }}=1.77 \Delta \phi_{a}, \\
& \left.\frac{\Delta L}{L}\right|_{\text {Sliding }}=0.51 \Delta \phi_{a} .
\end{aligned}
$$

A linear relation is also established between $\phi_{a}$ and the hydrostatic stress $\left(\sigma_{h}\right)$ inside the polymer volume where light will interact with the system (around the optical axis in Fig. 2a). As for the simplified analytical model, the hydrostatic stress $\left(\sigma_{h}\right)$ is found to be negligible compared with the bulk modulus of PDMS based polymers. Its contribution to the sensitivity of the sensors can therefore still be neglected, as in Section 2.1.1.

Finally, replacing Eq. (5) by Eq. (10) in the formalism of Section 2.1.1 yields the sensitivity of the sensors $\left(\Gamma_{\phi}\right)$ for both the bonded and the sliding models:

$$
\begin{gathered}
\left.\Gamma_{\phi}\right|_{\text {Bonded }}=1.77 \alpha+\beta \frac{\left(n_{a}-n_{p}\right)}{n_{p}}, \\
\left.\Gamma_{\phi}\right|_{\text {Sliding }}=0.51 \alpha+\beta \frac{\left(n_{a}-n_{p}\right)}{n_{p}} .
\end{gathered}
$$

In Eq. (11) the sliding model yields a deformation sensitivity $(0.51 \alpha)$ that is twice smaller that what was predicted $(1.00 \alpha)$ by the simplified analytical model. This result was expected since the polymer is now free to expand in two directions ( $x$ and $z$ ) rather than only one for the analytical model (similarly, a polymer that would be free to expand in all three dimensions would yield a $0.33 \alpha$ sensitivity).

Surprisingly, the bonded model yields a higher deformation sensitivity $(1.77 \alpha)$ than what is predicted by the simplified analytical model in Eqs. (9a) and (9b) (1.00 $\alpha$ ). This is because, in Fig. 2(b), the polymer is not free to expand in the microchannel, on each side of the optical cavity. Consequently, the polymer located in these regions expands towards the optical axis of the system, where the deformation is amplified (see Appendix A2). However, this behavior is found to depend strongly on the polymer Poisson's ratio. For $v<0.499$, the predicted sensitivity of the bonded model quickly 

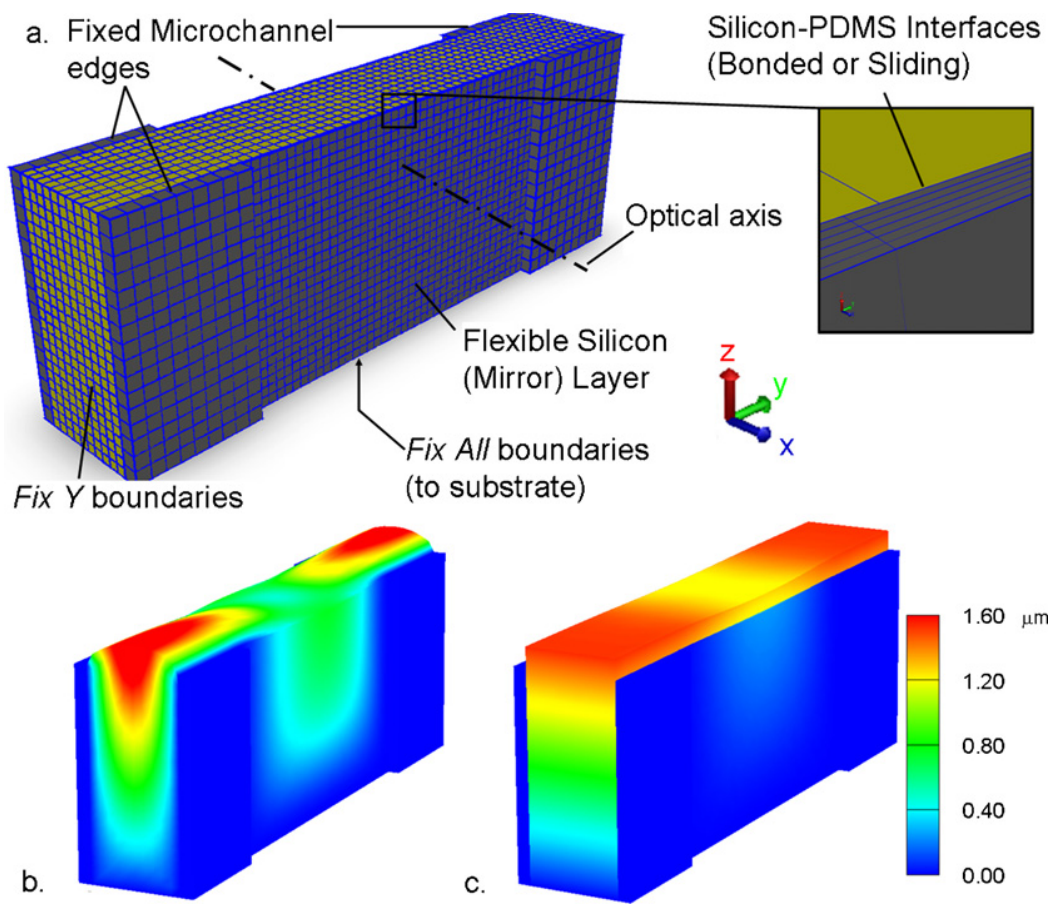

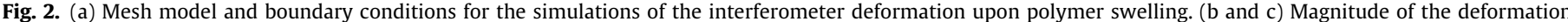

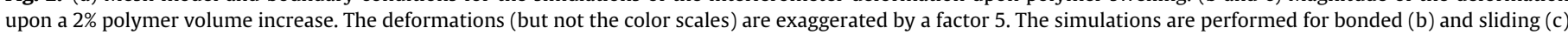
silicon-PDMS material interfaces.

reduces and essentially matches the sensitivity of the analytical model when $v<0.45$ (see Appendix A2).

\subsection{Experimental methodology}

A scanning electron microscope (SEM) photograph of a Fabry-Perot interferometer is presented in Fig. 3(b). This device has 3 silicon layers per mirror, compared to only two in the schematic view of Fig. 1. Both configurations were fabricated and tested for gas detection and, as predicted in Appendix A1, were found to respond similarly to deformations and refractive index changes. The interferometers are etched in silicon wafers using an inductively coupled plasma reactor (ICP180-100, Oxford Instruments Inc.). A two-mask soft lithography process is used to fabricate successively the smaller (multilayer mirrors) and the larger (optical fiber alignment grooves, microfluidic system) features [12].

The absorbent polymer (Fig. 3(c)) is inserted between the two mirrors of the interferometer using a monolithically integrated microfluidic system. A drop $(\sim 15-20 \mu \mathrm{L})$ of liquid pre-polymer mixture is placed in the leftmost reservoir of Fig. 3(a). The polymer flows by capillary forces in the microfluidic channel to fill the gap in the interferometer (Fig. 3(c)), and is then left to cure at room temperature for several hours. We did not determine the minimum required polymer curing time. Some devices required more than $12 \mathrm{~h}$ of curing for stable performance, so all devices were cured for more than a week.

Two different polymers were tested for the functionalization of the interferometers. Some devices were coated with commercial PDMS (Dow Corning ${ }^{\circledR}$ Sylgard 184), while the others were functionalized with a PDMS-polydiphenylsiloxane copolymer (PDMS-PDPS). The copolymer was prepared by a procedure similar to the one previously reported [13], but using different mixture proportions (10\% polydiphenylsiloxane (PDPS) mole fraction, $3 \%$ titanium tetraisopropoxide cross-linker mole fraction).

The functionalized devices were tested for the detection of two different vapor phase analytes at room temperature and atmospheric pressure. To produce different analyte concentrations, saturated vapor (10,720 ppm for m-xylene, $125,340 \mathrm{ppm}$ for cyclohexane [13]) was first generated by bubbling nitrogen through a wash bottle containing the analyte in liquid phase. A two-channel mixing flowmeter (Praxair PRXFM-4621(22)) was then used to dilute back the saturated vapor with pure nitrogen, in order to lower analyte concentration. The first channel of the flowmeter (nitrogen) uses a tantalum bead float, while the second channel (saturated analyte vapor) uses a glass float. The flowmeter and gas bubbling assembly are shown in Fig. 4(a). A gas cap (Fig. 4(b)) was lowered over the sensor to allow analyte vapor to disperse evenly over the chip. The complete setup was housed inside a laminar flow hood. Cyclohexane and $\mathrm{m}$-xylene were chosen as the analytes to allow comparison with previous work using similar polymers $[13,18]$.

Upon gas exposure, the interferometer resonance wavelength was tracked in real time by continuously scanning a tunable external-cavity diode laser source (Ando AQ4320D). The laser was interfaced with the interferometer through conventional singlemode optical fibers (Corning ${ }^{\circledR}$ SMF-28), which were cleaved and inserted in the optical fiber alignment grooves (Fig. 3). The transmission spectrum was recorded at $30 \mathrm{~s}$ intervals using an InGaAs photodiode detector (Thorlabs D400FC) and a lock-in amplifier (Stanford Research Systems SRS844 RFCA). The voltage output of the lock-in amplifier was then collected using a 16-bit data acquisition USB device (Measurements Computing PMD 1608 FS) and custom-made LabView (National Instruments) software. Amplitude noise was minimized numerically in each recorded spectrum using a zero-phase shift, forward and reverse digital convolution filter (Matlab ${ }^{\circledR}$ “FILTFILT" function). A $1 \mathrm{~nm}$ wide Gaussian distribution $(\sigma=0.2 \mathrm{~nm})$ was used as the filter. The exact resonance wavelength position was then determined for each spectrum by fitting a 4th order polynomial to the top half of the filtered resonance peaks.Results and discussion

The response of a PDMS-coated interferometer to various mxylene and cyclohexane vapor concentrations is presented in Fig. 5. 

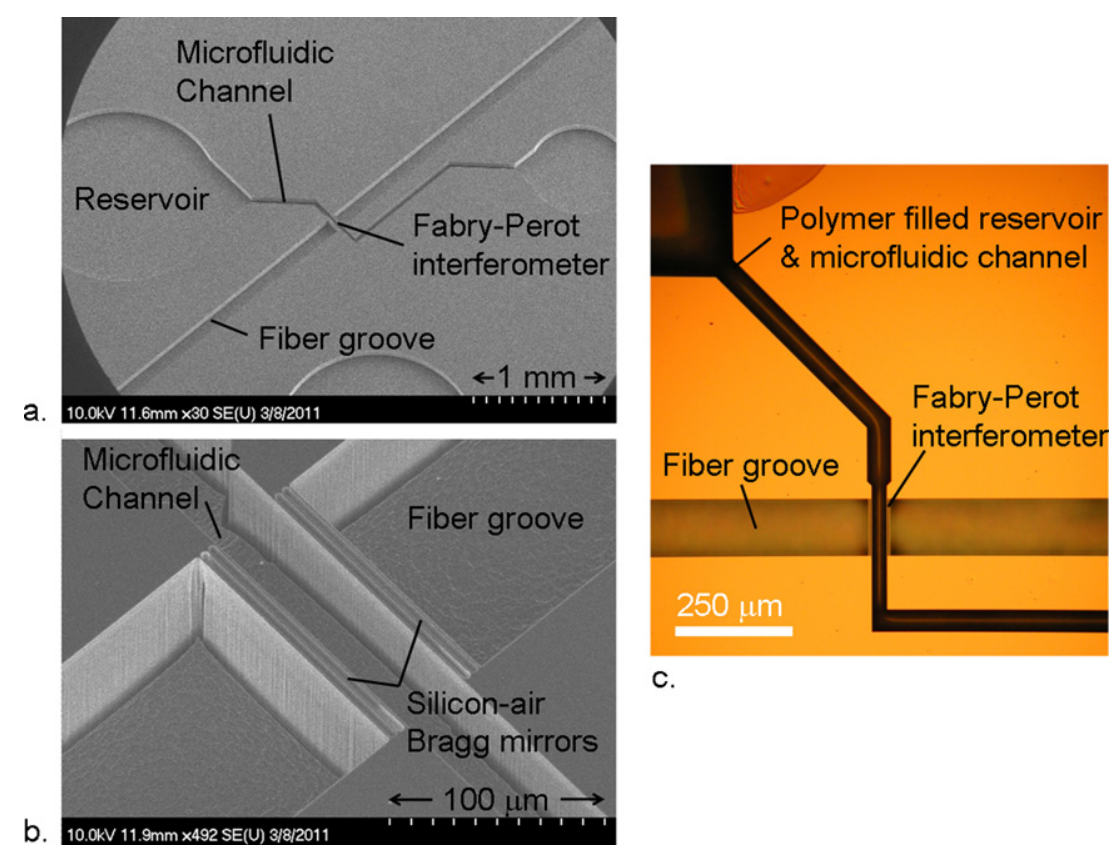

c.

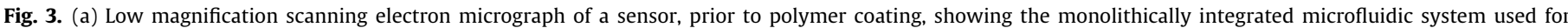

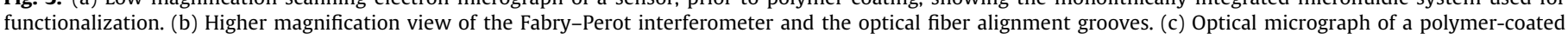
device.

As expected, in Fig. 5(a), the resonance peak position is found to increase upon exposition to $\mathrm{m}$-xylene, before returning to its initial value when the gas flow is switched back to pure nitrogen. In Fig. 5(b) and (c), this shift of the resonance wavelength is reported, as a function of time, for various concentrations of m-xylene and cyclohexane. The response to $34 \mathrm{ppm} \mathrm{m}$-xylene, the lowest concentration reachable with our flowmeter, is clearly visible. A limit of detection below this value is therefore expected.

The resonance wavelength increase $\left(\Delta \lambda_{\text {Res }}(t)\right)$ follows an essentially exponential pattern (i.e.: $\Delta \lambda_{\operatorname{Res}}(t) \propto 1-\mathrm{e}^{-t / \tau}$ ) with a time constant $(\tau)$ that differs for each polymer-analyte combination. In PDMS, the response time is found to be almost 4 times faster for cyclohexane ( $\tau=40 \mathrm{~s}$ ) than for $\mathrm{m}$-xylene $(\tau=150 \mathrm{~s})$. This faster response time however occurs at the expense of an approximately 4 time lower sensitivity to cyclohexane than to m-xylene.

This trade-off between sensitivity and response time is directly related to the partition coefficient $\left(K_{p-a}\right)$ of each polymer-analyte combination. Higher $K_{p-a}$ values yield higher absorbed volume fractions $\left(\phi_{a}\right)$ and therefore higher sensitivities. However, higher $K_{p-a}$ also yield higher retention times [19] (i.e. lower diffusion constants), which increase the time required for the analyte to diffuse completely inside the $80 \mu \mathrm{m}$ deep polymer-filled channel.

In Fig. 6 , the resonance wavelength shift $\left(\Delta \lambda_{\text {Res }}\right)$ for the different $m$-xylene vapor exposures is compared with the analytical model (Eq. (9b)), and with the finite element (Eq. (11)) bonded and sliding models. The expected response due only to refractive index variations is also included to highlight the influence of mechanical deformations on the sensitivity (a control experiment using undeformable mirrors was also carried to confirm the influence of refractive index variations only. See Appendix A3). The absorbed volume fraction of analyte $\left(\phi_{a}\right)$ is converted (Eq. (3)) to v/v gas concentrations $\left(C_{\text {air }}\right)$ in Eqs. (9b) and (11), using a $K_{p-a}=2090$ partition coefficient for m-xylene between PDMS and air [19]. We also use $V_{m}=0.123 \mathrm{~L} / \mathrm{mol}$, and the reported [13] refractive indices of PDMS $\left(n_{p}=1.3959\right)$ and xylenes $\left(n_{a}=1.4802\right)$ at $\lambda=1550 \mathrm{~nm}$.

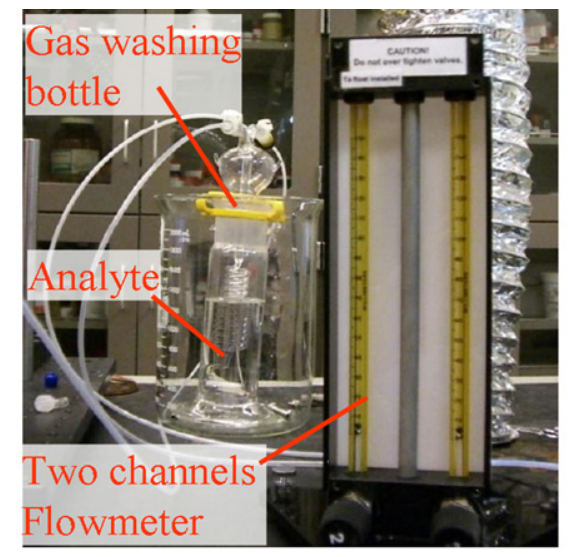

a.

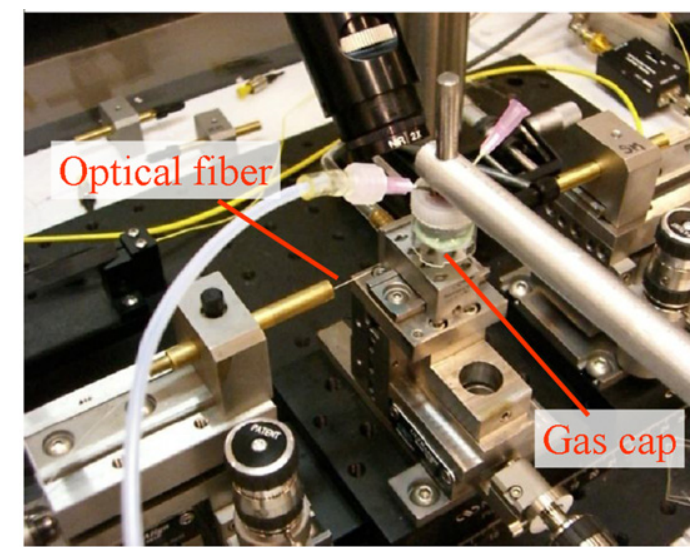

b.

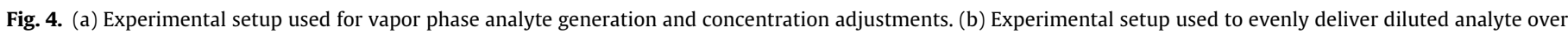
the sensor chip (which will eventually be positioned under the gas cap but is not shown here). 

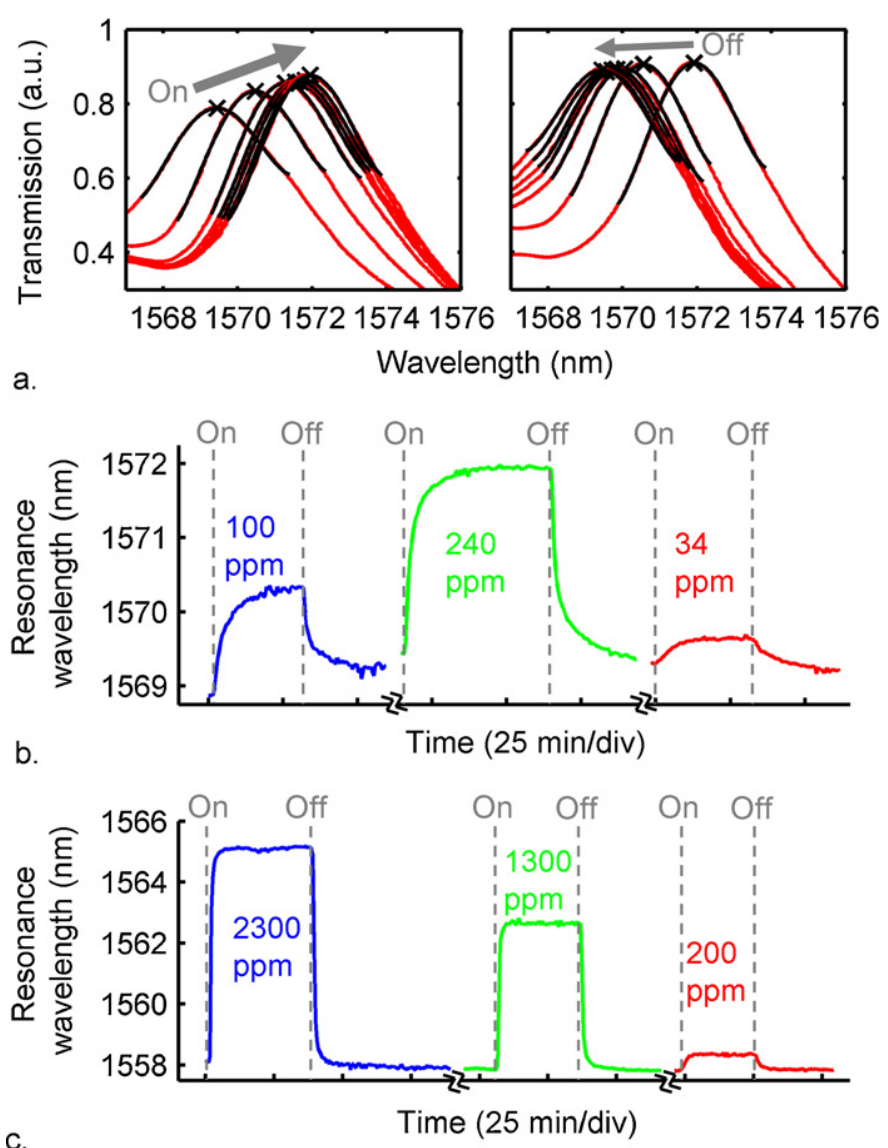

Fig. 5. (a) Measured transmission spectra of a PDMS-coated deformable FPI sensor upon exposure to a $240 \mathrm{ppm} \mathrm{m}$-xylene vapor concentration. The peak resonance wavelength ("X" marked) is measured by fitting a 4th order polynomial on top of the resonance peaks. Its position increases when gas flow is turned on (left) and then returns to its initial position (right) when the flow is switched back to pure nitrogen. (b and c) Resonance wavelength ( $\lambda_{\text {Res }}$ ) of a PDMS-coated interferometer over time. The "On" and "Off" markers designate the beginning and the end of exposure to the indicated concentrations of m-xylene (b) and cyclohexane (c).

As expected, the experimental results fall between the bonded and sliding models. The silicon-PDMS adhesion force appears to be important enough to yield a higher experimental sensitivity than the sliding model prediction. The sensitivity is, however, lower than the bonded model prediction. This could be an indication that PDMS does not bond perfectly with silicon, but is somewhat free to spatially reorganize itself upon expansion. Growing a thin layer of thermal silicon dioxide, prior to polymer coating, could be a way to increase the polymer-interferometer bonding strength and improve sensitivity. It is also possible that the bonding strength

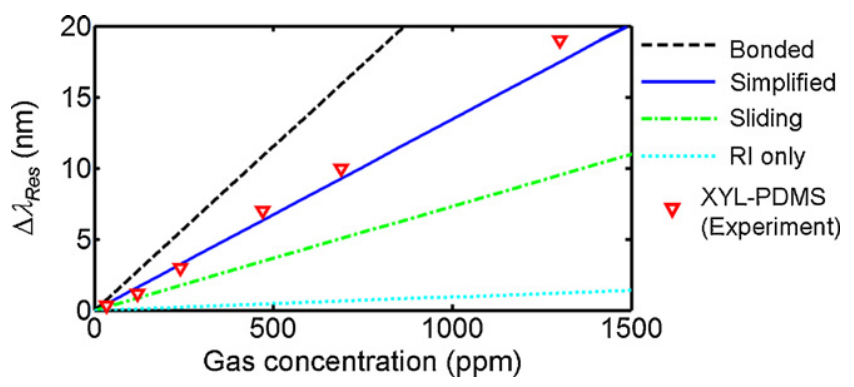

Fig. 6. Experimental and simulated (bonded, sliding and simplified analytical models) resonance wavelength shift ( $\Delta \lambda_{\text {Res }}$ ) as a function of m-xylene concentration. "RI only" designates the expected response due to refractive index variations only (i.e., neglecting mechanical deformations).

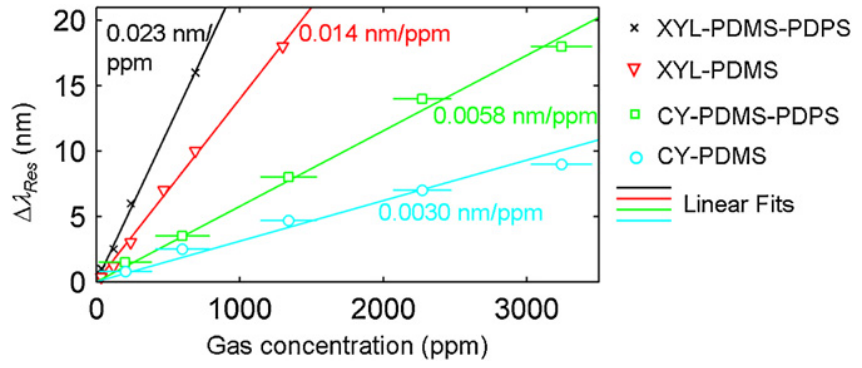

Fig. 7. Experimental response obtained for the four analyte-polymer combinations of m-xylene (XYL), cyclohexane (CY), PDMS, and the phenyl-doped copolymer (PDMS-PDPS). The linear fits are labelled by their corresponding sensitivities. The error bars are calculated form the repeatability ( $0.5 \%$ of full scale) of the two-channe flowmeter. As cyclohexane is more volatile than m-xylene, low concentration measurements suffer from greater uncertainty ( $m$-xylene error bars are too small to appear on graph).

is already high enough, but that finite element bonded sensitivity was overestimated. As discussed in Section 2.1.2 (see also Appendix A2), small variations of the PDMS Poisson's ratio tend to strongly affect the bonded model sensitivity, which rapidly reaches that of the simplified analytical model.

The simplified analytical model (Section 2.1.1) is found to reproduce our experimental results remarkably well. A relatively good correspondence was expected since, as shown in Eqs. (9b) and (11), the simplified analytical model yields an intermediate sensitivity between the two extreme possible cases (i.e., the bonded and sliding finite element models). Therefore, for other polymers having different mechanical properties, this model could most likely provide a useful estimate of the sensitivity without repeating time consuming finite element simulations. A perfect match with the experimental results, such as in Fig. 6, is however likely to be coincidence and should not be expected in all cases.

As discussed in Section 2.1.1, the simplified analytical model also describes the behavior of out-of-plane deformable FPI sensors [8-11]. Its correspondence with the current experimental results therefore indicates that the in-plane implementation of deformable FPI did not cause any significant sensitivity reduction compared to out-of-plane designs.

In Fig. 7, the experimental response is reported for m-xylenePDMS (XYL-PDMS) and also for the three other polymer-analyte combinations. The response to both analytes is essentially linear, within the repeatability of the flow meter $(0.5 \%$ of full scale for each channel). The highest sensitivity $(0.023 \mathrm{~nm} / \mathrm{ppm})$ is obtained for $\mathrm{m}$-xylene-PDMS-PDPS. This may be due to the addition of phenyl groups within the polymer, which could increase the polymer affinity for aromatic compounds, such as m-xylene. There are, however, other differences between the two polymers, including chain length and the type and degree of cross-linking, so other mechanical and chemical factors may also contribute to the sensitivity difference. The lowest sensitivity $(0.0030 \mathrm{~nm} / \mathrm{ppm})$ is obtained for cyclohexane $(\mathrm{CY})$ in PDMS, which may indicate that the partition coefficient $\left(K_{p-a}\right)$ is significantly lower for cyclohexane than for m-xylene. We did not find measurements of $K_{p-a}$ for CY-PDMS in the literature, but taking the linear temperature-programmed retention index of cyclohexane [20] (LTPRI $=650)$ and the relations reported in ref. [19], we expect $K_{p-a} \cong 320-350$. Using these values, the CY-PDMS experimental results are within the bonded and sliding models predictions.

The CY-PDMS-PDPS sensitivity $(0.0058 \mathrm{~nm} / \mathrm{ppm})$ is almost two times higher than the CY-PDMS response. This was not expected initially since cyclohexane is a non-polar analyte, and since the addition of phenyl groups to PDMS tends to increase its polarity [21]. It is possible that the phenyl groups also increase the Hildebrand solubility parameter of PDMS (initially $\delta=7.3 \mathrm{cal}^{1 / 2} \mathrm{~cm}^{-3 / 2}$ ) 
to a value closer to that of cyclohexane $\left(\delta=8.2 \mathrm{cal}^{1 / 2} \mathrm{~cm}^{-3 / 2}\right)[1]$. This phenomenon was previously reported [21,22] and could explain a higher $K_{p-a}$ for CY-PDMS-PDPS than for CY-PDMS. However, as noted above, there are other differences between the films that may also contribute to the observed $K_{p-a}$ difference, including chain length and degree of cross-linking. Moreover, those differences may also affect the polymer mechanical properties, which could influence the sensitivity.

In Fig. 7, PDMS-PDPS showed a 64\% increase in sensitivity to $\mathrm{m}$-xylene and a 93\% increase in sensitivity to cyclohexane as compared to PDMS. Having distinct sensitivity differences for each analyte could be useful for the development of artificial olfaction systems. Distinct sensitivity differences were exploited previously for artificial olfaction with other micromechanical sensors, such as cantilevered sensors [23], quartz crystal microbalances (QCM), surface acoustic wave (SAW) sensors [24,25], and out-of-plane deformable FPI sensors [10]. As the sensitivities of our sensors match those of out-of-plane FPIs (see discussion related to Fig. 6), it is likely that exploiting distinct sensitivity differences with our sensors could also allow specific detection.

For micromechanical sensors, the sensor limit of detection(LOD) is usually defined as the analyte concentration that produces a response corresponding to 3 times the standard deviation (std) of the background noise signal $[23,26]$. We calculated a std $=0.012 \mathrm{~nm}$ amplitude noise from the traces of Fig. 5(b and c), and also from the trace of a PDMS-PDPS coated device. Using the sensitivities of the PDMS-PDPS coated devices given in Fig. 7, we therefore expect LODs of $1.6 \mathrm{ppm} \mathrm{m}$-xylene and $6.3 \mathrm{ppm}$ cyclohexane. For comparison, previously reported LODs of QCM [26] and SAW [27] devices to xylenes vapors are on the same order of magnitude, at respectively $1.2 \mathrm{ppm}$ and $3.5 \mathrm{ppm}$. The reported devices could therefore potentially compete with these technologies, which already find applications in artificial olfaction systems [24,25].

The reported LOD is however more than one order of magnitude larger than what was reported using out-of-plane FPIs [10]. However, as discussed above, the resonance wavelength sensitivity of the reported devices, and of out-of-plane FPIs, are similar (see discussion related to Fig. 6). The significantly better LOD of out-of-plane devices is indeed not achieved through higher device sensitivities, but through optimization of a free-space optical interrogation setup [10] (as opposed, in our case, to passively aligned optical fibers). The reported in-plane devices, although less precise in term of LOD, could therefore still be advantageous in situations that would prevent on-site free-space alignment (e.g. field deployment for industrial artificial olfaction).

\section{Conclusions}

We reported a chemical sensor based on in-plane silicon Fabry-Perot interferometers functionalized with PDMS-based polymers. Upon analyte absorption, polymer swelling and refractive index variations were shown to induce strong shifts of the interferometer resonance wavelengths. Mechanical deformations were demonstrated to be the dominant sensing mechanism, in accordance with analytical and finite element models. The proposed analytical model is also expected to describe accurately the behavior of out-of-plane FPIs [8-11], and could therefore be useful for sensors relying on such optical configuration.

Fabry-Perot interferometers were functionalized with two different polymers (PDMS and PDMS-PDPS) and were used to detect and quantify two volatile organic compounds (m-xylene and cyclohexane). PDMS-PDPS devices were found to be $64 \%$ more sensitive to m-xylene and $93 \%$ more sensitive to cyclohexane than PDMS devices. Such distinct sensitivity differences, between sensor coatings, are promising for the development of artificial olfaction systems. Moreover, the expected limits of detection of the current proof-of-concept devices (1.6 ppm m-xylene, $6.3 \mathrm{ppm}$ cyclohexane) are already on the same order of magnitude as achieved with micromechanical sensing technologies used for artificial olfaction.

Compared with other types of micromechanical sensors, a possible advantage of deformable in-plane FPI sensors is their potential for field deployment and remote interrogation. The proposed sensor does not need any local source of energy to operate. Sensor heads could be deployed in industrial workplaces or for environmental monitoring, and would be linked to an interrogation system only through passively aligned single mode optical fibers. Using a $1 \times N$ optical switch would also allow the readout of multiple sensors (for various locations and/or for various polymers) using a single tunable laser and photodetector.

Compared with previously reported out-of-plane FPIs [8-11], the main distinctive feature of the proposed in-plane sensors is integrability. The in-plane configuration allowed monolithic integration of microfluidic systems, which simplified parallel functionalization of multiple interferometers with different polymers. Integrated fiber alignment grooves also allowed passive alignment of optical fibers, facilitating remote interrogation by avoiding the need for on-site free-space alignment. Finally, successful interfacing of swellable polymers with a silicon optical microsystem is, by itself, an interesting achievement. Such hybrid integration could allow great flexibility in the design of other interferometric, micromechanical, or even electromechanical systems that could be used to maximize sensitivity to polymer swelling.

\section{Acknowledgement}

This work was supported by the National Science and Engineering Research Council of Canada (NSERC).

\section{Appendix A. Supplementary data}

Supplementary data associated with this article can be found, in the online version, at http://dx.doi.org/10.1016/j.snb.2013.02.016.

\section{References}

[1] J.N. Lee, C. Park, G.M. Whitesides, Solvent compatibility of poly(dimethylsiloxane)-based microfluidic devices, Analytical Chemistry 75 (2003) 6544-6554.

[2] X. Liu, X. Zhang, J. Cong, J. Xu, K. Chen, Demonstration of etched cladding fiber Bragg grating-based sensors with hydrogel coating, Sensors and Actuators B: Chemical 96 (2003) 468-472.

[3] R.M. López, V.V. Spirin, M.G. Shlyagin, S.V. Miridonov, G. Beltrán, E.A. Kuzin, A Márquez Lucero, Coherent optical frequency domain reflectometry for interrogation of bend-based fiber optic hydrocarbon sensors, Optical Fiber Technology 10 (2004) 79-90.

[4] T.L. Yeo, T. Sun, K.T.V. Grattan, D. Parry, R. Lade, B.D. Powell, Characterisation of a polymer-coated fibre Bragg grating sensor for relative humidity sensing, Sensors and Actuators B: Chemical 110 (2005) 148-156.

[5] A. Convertino, A. Capobianchi, A. Valentini, E.N.M. Cirillo, A new approach to organic solvent detection: high-reflectivity bragg reflectors based on a gold nanoparticle/teflon-like composite material, Advanced Materials 15 (2003) 1103-1105.

[6] Y. Kang, J.J. Walish, T. Gorishnyy, E.L. Thomas, Broad-wavelength-range chemically tunable block-copolymer photonic gels, Nature Materials 6 (2007) 957-960.

[7] J.L. Martiǐnez-Hurtado, C.A.B. Davidson, J. Blyth, C.R. Lowe, Holographic detection of hydrocarbon gases and other volatile organic compounds, Langmuir 26 (2010) 15694-15699.

[8] G. Gauglitz, A. Brecht, G. Kraus, W. Mahm, Chemical and biochemical sensors based on interferometry at thin (multi-) layers, Sensors and Actuators B: Chemical 11 (1993) 21-27.

[9] C. Martínez-Hipatl, S. Muñoz-Aguirre, G. Beltrán-Pérez, J. Castillo-Mixcóatl, J. Rivera-De la Rosa, Detection of volatile organic compounds by an interferometric sensor, Sensors and Actuators B: Chemical 147 (2010) 37-42.

[10] K. Reddy, Y. Guo, J. Liu, W. Lee, M.K. Khaing Oo, X. Fan, Rapid, sensitive, and multiplexed on-chip optical sensors for micro-gas chromatography, Lab Chip 12 (2012) 901-905.

[11] F.R. Aussenegg, H. Brunner, A. Leitner, C. Lobmaier, T. Schalkhammer, F. Pittner, The metal island coated swelling polymer over mirror system (MICSPOMS): a 
new principle for measuring ionic strength, Sensors and Actuators B: Chemical 29 (1995) 204-209.

[12] R. St-Gelais, A. Poulin, Y.-A. Peter, Advances in modeling, design, and fabrication of deep-etched multilayer resonators, Journal of Lightwave Technology 30 (2012) 1900-1908.

[13] J. Barnes, M. Dreher, K. Plett, R.S. Brown, C.M. Crudden, H.-P. Loock, Chemical sensor based on a long-period fibre grating modified by a functionalized polydimethylsiloxane coating, Analyst 133 (2008) 1541-1549.

[14] T.M. Aminabhavi, Use of mixing rules in the analysis of data for binary liquid mixtures, Journal of Chemical and Engineering Data 29 (1984) 54-55.

[15] P. Brocos, A. Pineiro, R. Bravo, A. Amigo, Refractive indices, molar volumes and molar refractions of binary liquid mixtures: concepts and correlations, Physical Chemistry Chemical Physics 5 (2003) 550-557.

[16] J.E. Mark, Polymer Data Handbook, Oxford University Press, New York, 1999.

[17] F. Schneider, T. Fellner, J. Wilde, U. Wallrabe, Mechanical properties of silicones for MEMS, Journal of Micromechanics and Microengineering 18 (2008) 065008.

[18] J.A. Barnes, R.S. Brown, A.H. Cheung, M.A. Dreher, G. Mackey, H.-P. Loock, Chemical sensing using a polymer coated long-period fiber grating interrogated by ring-down spectroscopy, Sensors and Actuators B: Chemical 148 (2010) 221-226.

[19] P.A. Martos, A. Saraullo, J. Pawliszyn, Estimation of air/coating distribution coefficients for solid phase microextraction using retention indexes from linear temperature-programmed capillary gas chromatography. application to the sampling and analysis of total petroleum hydrocarbons in air, Analytical Chemistry 69 (1997) 402-408.

[20] Y. Guan, J. Kiraly, J.A. Rijks, Interactive retention index database for compound identification in temperature-programmed capillary gas chromatography, Journal of Chromatography 472 (1989) 129-143.

[21] İ. Yilgör, J. McGrath, Polysiloxane Containing Copolymers: A Survey of Recent Developments, in: Advances in Polymer Science, Springer, Berlin, Heidelberg, 1988, pp. 1-87.

[22] J.-C Huang, Probe dependency of segmental interaction parameters in copolymers by inverse gas chromatography, Journal of Applied Polymer Science 119 (2011) 719-725.

[23] D. Lange, C. Hagleitner, A. Hierlemann, O. Brand, H. Baltes, Complementary metal oxide semiconductor cantilever arrays on a single chip: mass-sensitive detection of volatile organic compounds, Analytical Chemistry 74 (2002) 3084-3095.

[24] K. Arshak, E. Moore, G.M. Lyons, J. Harris, S. Clifford, A review of gas sensors employed in electronic nose applications, Sensor Review 24 (2004) 181-198.

[25] A. Wilson, M. Baietto, Applications and advances in electronic-nose technologies, Sensors 9 (2009) 5099-5148.

[26] A. Mirmohseni, V. Hassanzadeh, Application of polymer-coated quartz crystal microbalance (QCM) as a sensor for BTEX compounds vapors, Journal of Applied Polymer Science 79 (2001) 1062-1066.

[27] N. Barié, M. Rapp, H.J. Ache, UV crosslinked polysiloxanes as new coating materials for SAW devices with high long-term stability, Sensors and Actuators B: Chemical 46 (1998) 97-103.

\section{Biographies}

Raphael St-Gelais received the B.Eng. (2007) and Ph.D. (2012) degrees in engineering physics from Polytechnique Montreal (Montreal, QC, Canada). In 2007, he was an engineering intern with Dalsa Semiconductor (Bromont, QC, Canada), before joining the Microphotonics Laboratory (Polytechnique Montreal) in 2008 for his graduate studies. He is currently a postdoctoral associate with the Cornell Nanophotonics Group (Cornell University, Ithaca, NY). His research interests include optical sensors, optical microelectromechanical systems, integrated optics, nanophotonics, and optofluidics.

Gillian Mackey received her B.Sc. degree in environmental chemistry from Queen's University in 2009. She is currently working towards her Ph.D. in chemistry at Queen's University under the supervision of Dr. R. Stephen Brown. Her research interests focus on environmental analytical chemistry and her current project involves the development of new sensors for pathogens in contaminated drinking water.

John Saunders is currently completing a Ph.D. degree in the Department of Chemistry at Queen's University under the supervision of Dr. Hans-Peter Loock. He completed his B.Sc. in Chemistry at Acadia University in 2009 working on his honour's project with Dr. Anthony Zhaoguo Tong. He now studies the use of polymers and periodic materials with micro-optical devices for chemical refractive index sensing of volatile organic compounds and aqueous heavy metals.
Jingjing Zhou is an Assistant Professor at Hubei University of Technology, China and a Post Doctoral Research Fellow at Queen's University, Canada. She earned her Bachelor's Degree from Central China Normal Univeristy in 2002 and Doctorate from Wuhan University, China with a dissertation on novel sol-gel solid phase microextraction coatings in 2007. Her current postdoctoral research is focused on polymer optical sensors and their applications in water quality monitoring and pollution control.

Antoine Leblanc-Hotte ing. Jr., was born in Quebec, Canada in 1987. He received his B.Sc. degree in engineering physics from École Polytechnique de Montréal in 2010. He is a Ph.D. student in the Microphotonics Laboratory at École Polytechnique de Montréal. His research is focused on optofluidic biomedical microsystems in silicon for single cell analysis

Alexandre Poulin received the M.Sc. degree in engineering physics from École Polytechnique de Montréal, Canada, in 2012. During his Master Degree he completed a research internship at École Polytechnique Fédérale de Lausanne, Switzerland and Federal Office of Metrology METAS, Switzerland, with the support of a FQRNT international internship award. He is currently pursuing the Ph.D. degree in Microsystems and microelectronics from the École Polytechnique Fédérale de Lausanne, Switzerland. His current research interests include microelectromechanica systems, microphotonics and electroactive polymers for development of sensors and actuators.

Jack A. Barnes is a Senior Research Associate in the Department of Chemistry at Queen's University. His research background includes chemical dynamics, molecular beam laser spectroscopy and the application of spectroscopy to environmenta monitoring. His undergraduate degree was obtained at McMaster University, followed by a Ph.D. at the University of Toronto. Postdoctoral work was conducted at the Centre for Molecular Beams and Laser Chemistry at the University of Waterloo. Further work in laser spectroscopy was conducted at the University of Victoria before coming to Queen's University. His current research interests involve the use of fiber optic detectors and microresonator structures in chemical sensing.

Hans-Peter Loock is a Professor of Chemistry with an interest in the development of fiber-optic and micro-photonic sensors for absorption, refractive index, strain, and fluorescence. His group specializes on the chemical detection in very small sample volumes. Together with his group and with collaborators he has developed optical detection methods that allow for Chemical Quantitation but do not require accurate intensity measurements. He has obtained an Engineering Chemistry degree from the Technical University of Darmstadt (Germany) in 1991 and a Ph.D. in physica chemistry from the University of Victoria, Canada. After two years of postdoctoral work at the Steacie Institute for Molecular Sciences (NRC, OT) he joined Queen's University in 1999.

R. Stephen Brown was born in Nova Scotia, Canada, and received his B.Sc. in Chemistry at Dalhousie University in 1985. He then moved to the University of Toronto to complete M.Sc. and Ph.D. degrees in the group of Ulrich Krull, completing the latter in 1992. He then joined the National Research Council of Canada's Biotechnology Research Institute in Montreal, and worked there in the Environmental Biotechnology division until 1996. His work was mainly in developing chemical sensors and other analysis methods for various organic contaminants in water. In 1996 he moved to Queen's University, where he is jointly in the Chemistry Department and the School of Environmental Studies. His group invented a new water monitoring technology in 2001, and he co-founded a start-up company called Pathogen Detec tion Systems in 2003 to commercialize the technology. The start-up was acquired in 2009 by the global water company Veolia, and commercialization is now progressing on a global level under the brand Endetec. Dr. Brown continues to work for Queen's and with Veolia on research to develop new sensors and other water monitoring technologies.

Yves-Alain Peter received the M.Sc. degree in Physics and the Dr.Sc. degree in Sciences from the University of Neuchâtel, Neuchâtel, Switzerland, in 1994 and 2001, respectively. In 1995, he was a Research Associate in the Department of Medical Radiobiology, Paul Scherrer Institute, Switzerland. During 1995-2001, he was a Graduate Research Assistant with the Applied Optics Group, Institute of Microtechnology, University of Neuchâtel. From 2001 to 2003, he was a Postdoctoral Researcher with the Microphotonics Group, Stanford University, Stanford, CA. From 2003 to 2004, he was an R\&D Engineer and Project Leader with the Swiss Center for Electronics and Microtechnology, Switzerland. He is currently an Associate Professor in the Department of Engineering Physics, Ecole Polytechnique de Montréal, Montréal, QC, Canada. His research interests include microphotonics and microoptoelectromechanical systems. 